\title{
The Immunoexpression of YAP1 and LATS1 Proteins in Clear Cell Renal Cell Carcinoma: Impact on Patients' Survival
}

\author{
J. Godlewski $(D),{ }^{1}$ J. Kiezun, ${ }^{1}$ B. E. Krazinski $(D),{ }^{1}$ Z. Kozielec, ${ }^{2}$ \\ P. M. Wierzbicki, ${ }^{3}$ and Z. Kmiec ${ }^{1,3}$ \\ ${ }^{1}$ Department of Human Histology and Embryology, School of Medicine, Collegium Medicum, \\ University of Warmia and Mazury in Olsztyn, Olsztyn, Poland \\ ${ }^{2}$ Department of Pathomorphology, School of Medicine, Collegium Medicum, \\ University of Warmia and Mazury in Olsztyn, Olsztyn, Poland \\ ${ }^{3}$ Department of Histology, Medical University of Gdansk, Gdansk, Poland \\ Correspondence should be addressed to J. Godlewski; janusz350@poczta.onet.pl
}

Received 28 December 2017; Accepted 5 March 2018; Published 3 April 2018

Academic Editor: Stefan Zastrow

Copyright (C) 2018 J. Godlewski et al. This is an open access article distributed under the Creative Commons Attribution License, which permits unrestricted use, distribution, and reproduction in any medium, provided the original work is properly cited.

The aim of the study was to determine by immunohistochemistry cellular localization and immunoreactivity levels of YAP1 and LATS1 proteins in paired sections of tumor and unchanged renal tissues of 54 clear cell renal cell carcinoma (ccRCC) patients. Associations between clinical-pathological and overall survival (OS; median follow-up was 40.6 months) data of patients and YAP1 and LATS1 immunoreactivity were analyzed by uni- and multivariate Cox regression model and log-rank test. YAP1 immunoreactivity was found in the nuclei of tumor cells in $64.8 \%$ of ccRCC patients, whereas only $24.1 \%$ of tumors revealed cytoplasmic YAP1 expression. LATS1 immunoexpression was observed only in the cytoplasm of tumor cells in $59.3 \%$ of patients. LATS1 immunoreactivity in cancer cells negatively correlated with the size of primary tumor. The overall YAP1 immunoreactivity did not correlate with clinical-pathological data of patients. However, the subgroup of ccRCC patients who presented with cytoplasmic YAP1 immunoexpression had significantly shorter OS (median $=26.8$ months) than patients without cytoplasmic YAP1 expression (median undefined). Multivariate Cox analysis revealed that increased cytoplasmic YAP1 $(\mathrm{HR}=4.53)$ and decreased LATS1 immunoreactivity levels $(\mathrm{HR}=0.90)$ were associated with worse prognosis, being independent prognostic factors. These results suggest that YAP1 and LATS1 can be considered as new prognostic factors in ccRCC.

\section{Introduction}

Renal cell carcinoma (RCC) is the most common type of cancer of the urinary tract. According to the latest worldwide registry data, 337,860 new cases $(123,936$ women and 213,924 men) with the total mortality of 143,406 cases $(52,604$ and 90,802 resp.) were reported in 2012 [1]. The RCC encompasses a group of heterogeneous tumors which originate from the renal tubular epithelial cells. The most frequent type of RCC is the clear cell renal cell carcinoma (ccRCC). It originates from the proximal tubular epithelium and is characterized by the worst clinical course and prognosis among other RCC types [2]. The genetic and epigenetic background of alterations that occur during development and progression of ccRCC has not been fully elucidated so far.
Yes-associated protein 1 (YAP1) may be considered as one of the oncoproteins that play an important role in ccRCC pathogenesis, since deregulation of this gene (either at mRNA or protein level) was associated with progression of other malignancies [3-5]. YAP1 gene is located at 11q22 chromosome and this locus is amplified in many tumors [6]. $Y A P 1$, as an oncogene, plays a role in cells' proliferation and furthermore it is also involved in other mechanisms of cancer progression such as epithelial-mesenchymal transition, cell migration, and formation of metastasis [7]. Moreover, numerous studies have shown higher YAP1 gene expression as well as higher level of YAP1 protein in numerous cancers such as non-small-cell lung, breast, colorectal, and liver cancers [8]. 
YAP1 protein is a transcriptional coactivator which does not contain a DNA-binding domain; however, it interacts with transcription factors such as TEA domain (TEAD1-4) proteins binding to genes' promoters. Such a functional complex, composed of YAP1 and TEAD1-4 proteins, promotes expression of genes which are associated with cellular progression and proliferation (e.g., CTGF, Cyr61, Myc, and Gli) $[9,10]$. During physiological conditions, YAP1/TEAD complex acts as the transcriptional effector of the Hippo pathway that during development affects organs' size by inhibition of cell proliferation and activation of apoptosis [11]. The crucial component of the Hippo pathway is serine/threonineprotein kinase 1 (LATS1) that forms Hippo core protein cassette (together with MST2 protein) $[12,13]$. Hippo pathway negatively regulates YAP1 translocation to nucleus via LATS1dependent phosphorylation of YAP1 which in turn binds to cytoplasmic 14-3-3 protein. As a consequence the complex YAP1/14-3-3 is targeted for proteasomal degradation [14]. The translocation of YAP1 from the cytoplasm to the nucleus is necessary for its function as transcriptional coactivator. In this way the Hippo pathway components control and may inhibit cellular growth. In our previous study we found at both mRNA and protein levels that LATS1 gene expression was downregulated and YAP1 expression upregulated in ccRCC tumor tissues in comparison to corresponding samples of unaltered kidney tissues [13]. However, the cellular localization and expression of both proteins by immunohistochemistry (IHC) was not performed, and the studies of other authors provided opposite findings $[15,16]$. Therefore, we decided to assess the immunoreactivity of LATS1 and YAP1 proteins within the cancer and normal kidney tissue of patients with ccRCC. The results of the IHC study were correlated with the clinical and pathological features of ccRCC patients. The postoperative follow-up was performed in order to evaluate the immunoexpression of the investigated proteins as possible risk factors of cancer progression and patients' survival.

\section{Material and Methods}

2.1. Clear Cell RCC Patients, Specimen Collection, and Ethics Statement. This study was carried out in accordance with the Declaration of Helsinki (1964). All procedures were approved by the Bioethics Committee for Scientific Research at the University of Warmia and Mazury in Olsztyn, (decision number 4/2010). Appropriate written informed consent regarding the use of tissue was obtained from each patient in the study. The fragment of postoperative tumor tissue and unchanged kidney tissue were obtained from 54 ccRCC patients (23 females and 31 males) with a mean age $64.07 \pm 9.10$, range 44-83 years, who underwent surgery at the Department of Oncological Surgery, Warmia and Mazury Oncological Center, Olsztyn, Poland, in the period between March 2010 and May 2014. None of the patients had suffered from a second neoplastic disease or other serious disease. The clinical characteristics and overall survival (OS) data of the patients were collected during the study and the median time of follow-up was 40.6 months. The tumor stage was characterized according to the TNM system (American Joint
Committee on Cancer) [17]. Hematoxylin and eosin- (H\&E-) stained sections of collected tumor and matching kidney specimens were evaluated by a pathologist to confirm their cancer or cancer-free phenotype, respectively. The degree of tumor malignancy was determined using the Fuhrman nuclear grading system [18]. The studied tissues were placed in $4 \%$ buffered formaldehyde, postfixed, dehydrated, embedded in paraffin, and cut into $5 \mu \mathrm{m}$ thick sections.

2.2. Immunohistochemistry. Immunohistochemical (IHC) analysis was performed as described previously by Godlewski et al. [20] with some modification. The sections were subjected to an antigen retrieval procedure by microwaving in Retrieval Solution Buffer $(\mathrm{pH}$ 6.0; Leica, Wetzlar, Germany), incubated with $3 \% \mathrm{H}_{2} \mathrm{O}_{2}$ in methanol for $30 \mathrm{~min}$, and then in commercial normal horse serum (Vector Laboratories, Inc., Burlingame, CA, USA) for $30 \mathrm{~min}$. The sections were incubated overnight at $4^{\circ} \mathrm{C}$ with rabbit polyclonal anti-human antibodies against YAP1 (diluted 1:800 in PBS; \#ab81183; Abcam, Cambridge, UK) or LATS1 (diluted 1: 800 in PBS; \#LS-C98952, LifeSpan Biosciences, Seattle, WA, USA) and then with secondary antibodies (commercially diluted; ImmPRESS Universal Reagent Anti-Mouse/Rabbit Ig; Vector Laboratories, Inc., Burlingame, CA, USA) for $30 \mathrm{~min}$. To visualize the immunoreaction, the sections were immersed in 3,3'-diaminobenzidine (DAB; Dako, Glostrup, Denmark) and counterstained in Mayer's hematoxylin. The tissue sections were dehydrated in ethanol, cleared in xylene, and mounted. The specificity of the IHC staining was determined by omitting the primary antibody and replacing it with the same dilution of horse serum. The labelled tissues were photographed using a XC-50 camera (Olympus Corporation, Tokyo, Japan) mounted on a light microscope (BX-41; Olympus Corporation). Concomitantly to IHC, the $\mathrm{H} \& \mathrm{E}$ staining was performed to assess tissue morphology.

2.3. Evaluation of Immunohistochemical Reactions. IHC reactions for YAP1 and LATS1 in ccRCC tumors and corresponding normal kidney tissue were evaluated by pathologist who was blinded to the patients' clinical data. The scoring system for YAP1 nuclear immunoreactivity was based on the percentage of YAP1-immunoreactive cells: 0 for negative, 1 for up to $25 \%, 2$ for up to $75 \%$, and 3 for more than $75 \%$ of YAP1immunoreactive cells. The intensity of YAP1 cytoplasmic IHC reactions was scored as follows: 0 for negative, 1 for weak, 2 for moderate, and 3 for strong. The scoring system for LATS1 cytoplasmic immunoreactivity was based on the percentage of LATS1-immunoreactive cells and the intensity of IHC reaction according to the immunoreactive score system (IRS) of Remmele and Stegner [19]. The scale is based on the percentage of cells showing positive reaction, 1 point: $1-10 \%$ cells, 2-points: $11-50 \%, 3$ points: $51-80 \%$, and 4 points: over $80 \%$ cells with positive reaction, as well as reaction intensity (0: no reaction, 1 low intensity reaction, 2 : moderate intensity reaction, and 3: intense reaction). The final score depended on both parameters, percentage of positive cells and intensity of the reaction, and ranged from 0 to 12 points.

2.4. Statistical Analysis. Statistical analysis was carried out using Prism 6 (GraphPad, La Jolla, CA, USA) and Statistica 


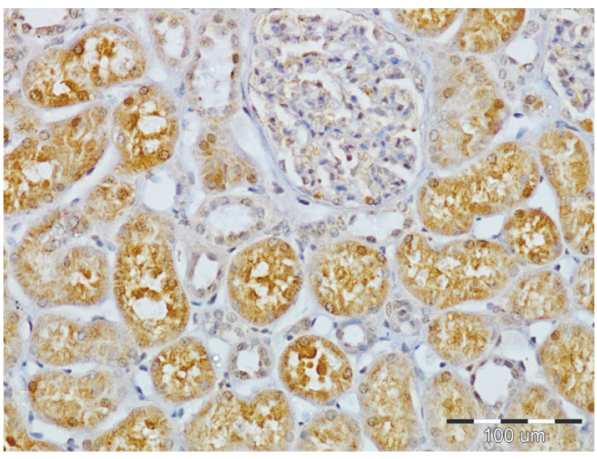

(a)

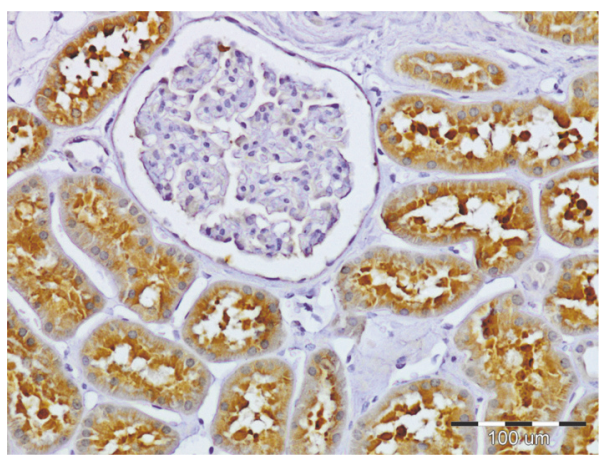

(c)

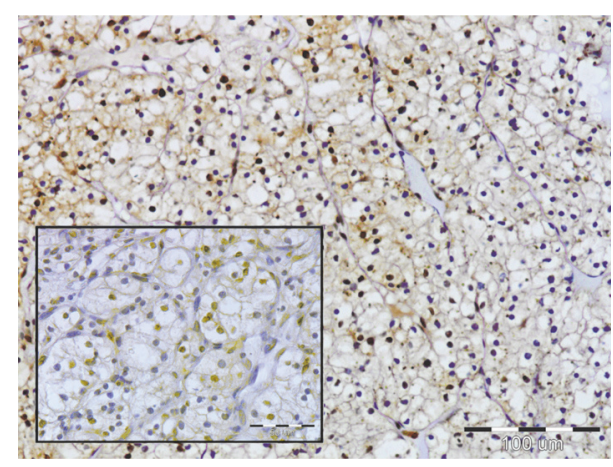

(b)

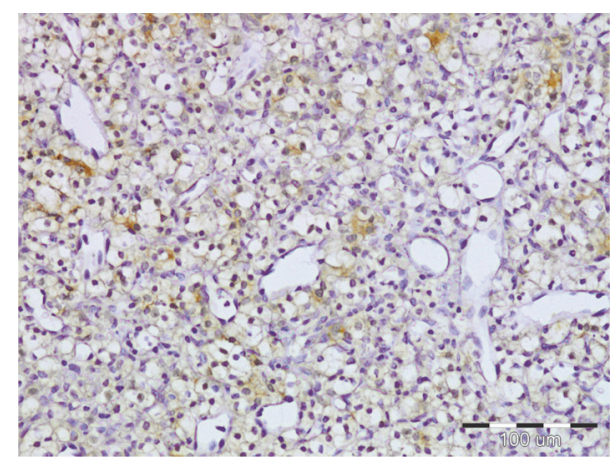

(d)

FIGURE 1: Evaluation of Yes-associated protein 1 (YAP1) and serine/threonine-protein kinase 1 (LATS1) expression levels in the sections of clear cell renal cell carcinoma (ccRCC) and unchanged kidney tissues by immunohistochemistry. In unchanged kidney cortex (a) strong YAP1 immunoreactivity was localized mainly in the cytoplasm of epithelial cells of proximal convoluted tubules (PCT). In ccRCC tumor cells YAP1 was expressed mainly in cell nuclei ((b) insert); however, weak to moderate YAP1 immunoreactivity was also observed in the cytoplasm of tumor cells in 13/54 ccRCC patients (b). Strong LATS1 immunoreactivity was present in the cytoplasm of PCT epithelium (c) and weak immunoreactivity in the cytoplasm of ccRCC cells (d), respectively. Magnifications: (a) $-(d) \times 200$; insert (b) $\times 300$.

13 (StatSoft, Tulsa, OK, USA) software. Significance of differences between expression levels of analyzed proteins in ccRCC cells and proximal convoluted tubules (PCT) epithelial cells was tested by the Wilcoxon matched-pairs signed rank test. Correlations between the immunoexpression of the studied proteins and between levels of protein expression and clinical-pathological data were evaluated using Fisher exact test and Spearman's correlation test. Patients' OS fractions were calculated and visualized according to the KaplanMeier method and the statistical significance of differences in survival between groups of patients was evaluated by log-rank test. The univariate and multivariate associations of clinicalpathological data with patients' survival and immunoreactivity were assessed using Cox proportional hazards regression model. Differences were considered statistically significant for $p<0.05$.

\section{Results}

3.1. Tumor ccRCC Cells Exhibit Altered YAP1 Immunoreactivity That Does Not Correlate with Clinical-Pathological Data of the Patients. YAP1 protein immunoreactivity was found in both ccRCC tumor and normal kidney sections. However, ccRCC cells exhibited predominantly nuclear YAP1 immunoreactivity (Figures 1(a) and 1(b), insert), whereas epithelial cells of the PCT were characterized by predominant cytoplasmic expression of YAP1 (Figure 1(a)). The nuclear immunoreactivity of YAP1 was moderate to strong in 35/54 $(64.8 \%)$ and absent or weak in 19/54 (35.2\%) of ccRCC specimens. In $75.6 \%$ of ccRCC patients YAP1 immunoreactivity was absent in the cytoplasm of tumor cells; however, in 13/54 (24.1\%) of ccRCC patients tumor cells exhibited weak to moderate cytoplasmic YAP1 immunoreactivity (Figure 1(b)). In comparison to the respective cellular compartments of PCT cells of normal kidney the immunoexpression of YAP1 was significantly increased in the nuclei (Figure 2(a)) and decreased in the cytoplasm (Figure 2(b)) of ccRCC tumor cells.

YAP1 nuclear and cytoplasmic expression levels in the tumor cells did not correlate with demographic and clinicalpathological data of patients with ccRCC (Table 1).

3.2. LATS1 Immunoreactivity Is Decreased in ccRCC Cells and Is Associated with a Size of the Primary Tumor. LATS1 immunoexpression was found only in the cytoplasm of tumor cells and PCT epithelium (Figures 1(c) and 1(d)). LATS1 immunoreactivity was significantly lower in ccRCC cells as compared to the PCT cells of normal kidney (Figure 3). 


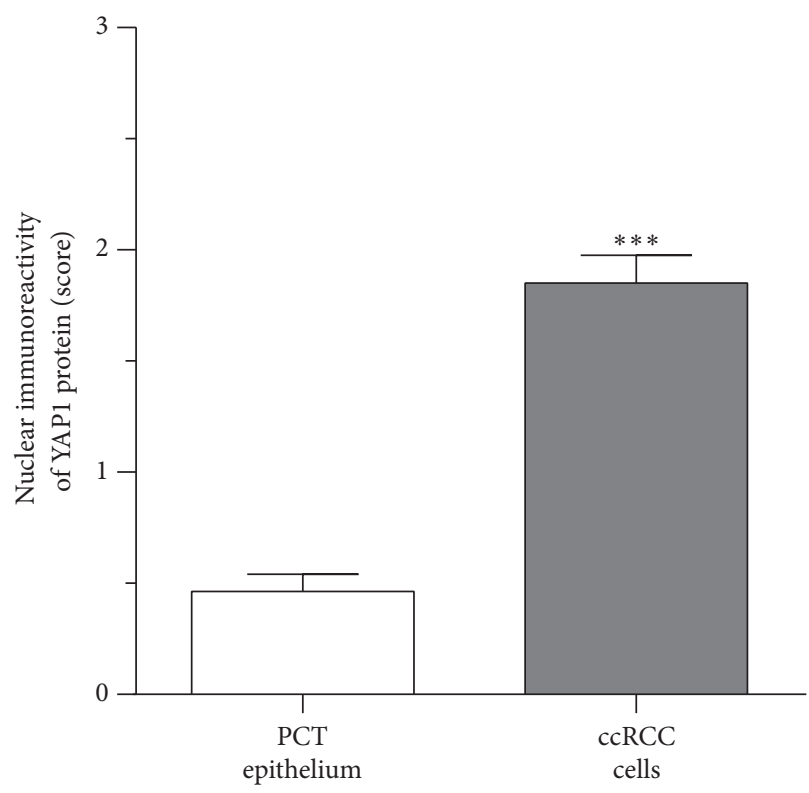

(a)

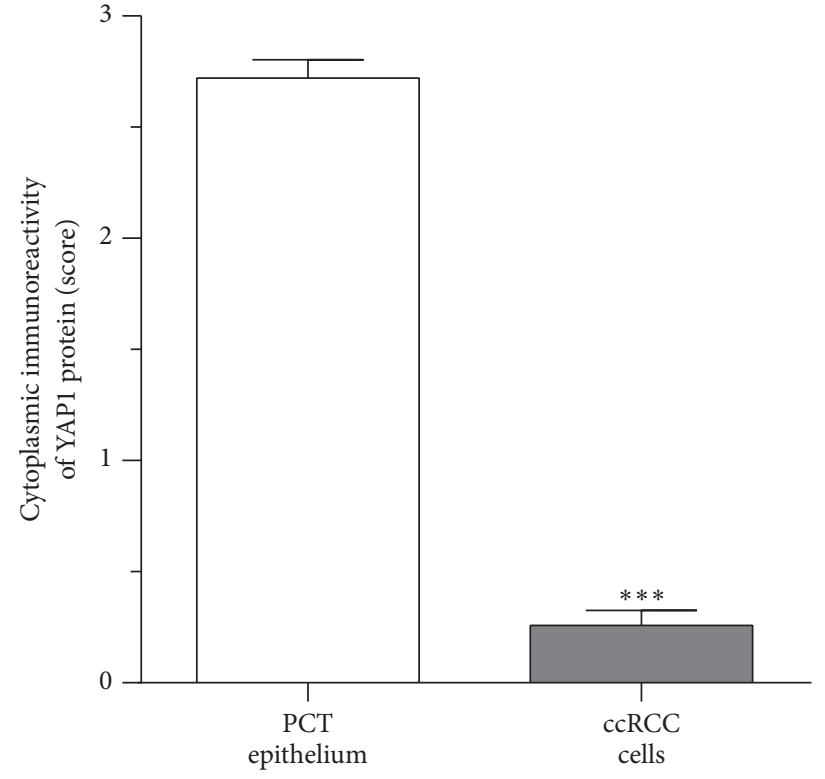

(b)

FIGURE 2: Comparison of Yes-associated protein 1 (YAP1) expression in the clear cell renal cell carcinoma (ccRCC) and normal kidney tissues assessed by immunohistochemistry. The levels of nuclear (a) and cytoplasmic (b) YAP1 immunoreactivity in the tumor cells are shown in relation to the levels of YAP1 immunoreactivity in the epithelial cells of proximal convoluted tubules (PCT) of normal kidney. Data are presented as the mean $\pm \operatorname{SEM}(n=54)$. $p$ values were calculated using the Wilcoxon matched-pair test. ${ }^{* * *} p<0.001$ (see Methods).

However, the intensity of LATS1 immunoreactivity varied between cancer cells since it was absent or weak in $22 / 54$ (40.7\%) and moderate to strong in 32/54 (59.3\%) of ccRCC patients.

LATS1 immunoexpression in cancer cells negatively correlated with the size of primary tumor (Table 2).

3.3. YAP1 Nuclear Immunoreactivity Is Associated with YAP1 and LATS1 Presence in the Cytoplasm of ccRCC Cells. We found negative correlation between YAP1 nuclear and cytoplasmic immunoreactivity levels in cancer cells $(r=-0.32$, $p=0.0166)$. Moreover, cytoplasmic LATS1 immunoreactivity was positively associated with the nuclear localization of YAP1 immunoexpression $(r=0.34, p=0.0127)$.

3.4. Overall Survival of ccRCC Patients Is Associated with the Immunoreactivity of YAP1 and LATS1 Proteins in ccRCC Tumor Cells. Kaplan-Meier plots presenting the ccRCC patients survival and immunoreactivity of investigated protein are demonstrated in Figure 4. Lower nuclear YAP1 presence was associated with shorter survival of ccRCC patients (median $=50.0$ months versus undefined for high nuclear YAP1 expression, Figure 4(a)). On the contrary, the subgroup of $25 \%$ of ccRCC patients in whom cytoplasmic presence of YAP1 in cancer cells was observed showed significantly shorter OS (median $=26.8$ months) than patients without cytoplasmic YAP1 presence (median undefined) (Figure 4(b)). Also decreased cytoplasmic LATS1 immunoreactivity was associated with significantly shorter survival (Figure 4(c)). Univariate Cox proportional hazards regression revealed that increased YAP1 and decreased LATS1 immunoreactivity levels in the cytoplasm of ccRCC cells were significantly associated with worse prognosis (Table 3). Multivariate regression analysis confirmed that the expression levels of YAP1 and LATS1 proteins $(\mathrm{HR}=4.53$ and $\mathrm{HR}=0.90$, resp.), along with the presence of distant metastases ( $\mathrm{HR}=$ 4.94), are independent prognostic factors in ccRCC (Table 3).

\section{Discussion}

YAP1 is an activator of TEAD1-4 transcription factors and it has been found that TEAD1-4 family members play important role in tumor progression via activating progressioninducing genes (CTGF, Cyr61, and Myc) $[9,10]$. The role of YAP1 in the stimulation of cell proliferation is crucial during organogenesis and this function may be inhibited by the Hippo pathway components by cytoplasmic sequestration of YAP1 after its phosphorylation by LATS1 kinase [11, 12]. The IHC studies published so far underline the role of YAP1 in the course of several types of cancers of the alimentary tract and demonstrate the correlation between higher expression of YAP1 protein, cancer development, and poor patients' prognosis [21-23].

Our IHC study revealed predominant YAP1 protein localization within nuclei of ccRCC cells in contrast to the predominantly cytoplasmic localization in the cells of normal kidney tissue. Moreover, nuclear YAP1 immunoreactivity was significantly higher in ccRCC cells than in the nuclei of proximal tubules of histologically unaltered kidney cortex. These observations are in concordance with our previously performed analysis of the YAP1 expression at the mRNA 
TABLE 1: Correlations between clinical-pathological characteristics of the patients with clear cell renal cell carcinoma (ccRCC) and YAP1 immunoreactivity in the cancer cells (determined by immunohistochemistry).

(a)

\begin{tabular}{|c|c|c|c|c|c|c|c|}
\hline \multirow{2}{*}{$\begin{array}{l}\text { Qualitative } \\
\text { parameters }\end{array}$} & \multirow{2}{*}{$\begin{array}{l}\text { Number of } \\
\text { patients }\end{array}$} & \multicolumn{3}{|c|}{$\begin{array}{l}\text { YAP1 nuclear immunoreactivity in ccRCC } \\
\text { cells (expressed as scores) }{ }^{*}\end{array}$} & \multicolumn{3}{|c|}{$\begin{array}{l}\text { YAP1 cytoplasmic immunoreactivity in } \\
\text { ccRCC cells (expressed as scores) }\end{array}$} \\
\hline & & $\begin{array}{c}0-1 \\
n(\%)\end{array}$ & $\begin{array}{c}2-3 \\
n(\%)\end{array}$ & $p$ value & $\begin{array}{c}0 \\
n(\%)\end{array}$ & $\begin{array}{c}1-2 \\
n(\%)\end{array}$ & $p$ value \\
\hline Total & 54 & $\begin{array}{c}19 \\
(35.2)\end{array}$ & $\begin{array}{c}35 \\
(64.8)\end{array}$ & \multirow{3}{*}{$1.0000^{\mathrm{a}}$} & $\begin{array}{c}41 \\
(75.9)\end{array}$ & $13(24.1)$ & \multirow{3}{*}{$1.0000^{\mathrm{a}}$} \\
\hline Men & 31 & $\begin{array}{c}11 \\
(35.5)\end{array}$ & $\begin{array}{c}20 \\
(64.5)\end{array}$ & & $\begin{array}{c}24 \\
(77.4)\end{array}$ & $7(22.6)$ & \\
\hline Women & 23 & $\begin{array}{c}8 \\
(34.8)\end{array}$ & $\begin{array}{c}15 \\
(65.2)\end{array}$ & & $\begin{array}{c}17 \\
(73.9)\end{array}$ & $\begin{array}{c}6 \\
(26.1)\end{array}$ & \\
\hline \multicolumn{8}{|l|}{$\begin{array}{l}\text { Depth of } \\
\text { invasion }\end{array}$} \\
\hline $\mathrm{T} 1+\mathrm{T} 2$ & 42 & $\begin{array}{c}13 \\
(31.0)\end{array}$ & $\begin{array}{c}29 \\
(69.0)\end{array}$ & \multirow{2}{*}{$0.3066^{\mathrm{a}}$} & $\begin{array}{c}30 \\
(71.4)\end{array}$ & $\begin{array}{c}12 \\
(28.6)\end{array}$ & \multirow{2}{*}{$0.2538^{\mathrm{a}}$} \\
\hline T3 & 12 & $\begin{array}{c}6 \\
(50.0)\end{array}$ & $\begin{array}{c}6 \\
(50.0)\end{array}$ & & $\begin{array}{c}11 \\
(91.7)\end{array}$ & $\begin{array}{c}1 \\
(8.3)\end{array}$ & \\
\hline \multicolumn{8}{|l|}{$\begin{array}{l}\text { Fuhrman } \\
\text { grade }\end{array}$} \\
\hline $\mathrm{G} 1+\mathrm{G} 2$ & 42 & $\begin{array}{c}12 \\
(28.6)\end{array}$ & $\begin{array}{c}30 \\
(71.4)\end{array}$ & \multirow{2}{*}{$0.0867^{\mathrm{a}}$} & $\begin{array}{c}34 \\
(81.0)\end{array}$ & $\begin{array}{c}8 \\
(19.0)\end{array}$ & \multirow{2}{*}{$0.1340^{\mathrm{a}}$} \\
\hline G3 & 12 & $\begin{array}{c}7 \\
(58.3)\end{array}$ & $\begin{array}{c}5 \\
(41.7)\end{array}$ & & $\begin{array}{c}7 \\
(58.3)\end{array}$ & $\begin{array}{c}5 \\
(41.7)\end{array}$ & \\
\hline \multicolumn{8}{|l|}{$\begin{array}{l}\text { Distant } \\
\text { metastases }\end{array}$} \\
\hline M0 & 47 & $\begin{array}{c}16 \\
(34.0)\end{array}$ & $\begin{array}{c}31 \\
(66.0)\end{array}$ & \multirow{2}{*}{$0.6866^{\mathrm{a}}$} & $\begin{array}{c}35 \\
(74.5)\end{array}$ & $\begin{array}{c}12 \\
(25.5)\end{array}$ & \multirow{2}{*}{$1.0000^{\mathrm{a}}$} \\
\hline M1 & 7 & $\begin{array}{c}3 \\
(42.9)\end{array}$ & $\begin{array}{c}4 \\
(57.1)\end{array}$ & & $\begin{array}{c}6 \\
(85.7) \\
\end{array}$ & $\begin{array}{c}1 \\
(14.3)\end{array}$ & \\
\hline
\end{tabular}

(b)

\begin{tabular}{lcccc}
\hline Quantitative parameters & $r$ & $p$ value & $r$ & $p$ value \\
\hline Age & -0.08 & $0.5494^{\mathrm{b}}$ & -0.01 & $0.9256^{\mathrm{b}}$ \\
Tumor size & -0.08 & $0.5544^{\mathrm{b}}$ & 0.06 & $0.6581^{\mathrm{b}}$ \\
\hline
\end{tabular}

${ }^{*}$ The scores evaluate percentage cells with YAP1 nuclear immunoreactivity. ${ }^{* *}$ The scores assess intensity of YAP1 cytoplasmic IHC reactions, both described in Methods. ${ }^{a}$ Fisher exact test. ${ }^{b}$ Spearman's correlation.

and protein levels by QPCR and Western blotting techniques, respectively. In that study we showed that YAP1 gene upregulation and worse clinical outcome of ccRCC patients as well as higher YAP1 protein expression in cancer tissue homogenates were associated with the clinical stage and pathomorphological features such as higher TNM and Fuhrman's stages [13].

In ccRCC the immunoreactivity of YAP1 has been studied by only few authors. Cao et al. found that $63.3 \%$ (19/30) of ccRCC patients exhibited positive YAP1 immunoexpression, whereas the positive rate of YAP protein expression in the normal tissues was $33.3 \%(10 / 30)$ [15]. In contrast to our findings, they observed correlation between the protein presence in cancer tissues, low differentiated type of cancer, and advance stage of the illness [15]. However, these authors did not differentiate patients in relation to the intensity of YAP1's nuclear or cytoplasmic immunoreactivity as presented in our study. On the contrary, Hu et al. reported lower YAP protein immunoreactivity in tissue microarrays of $75 \mathrm{ccRCC}$ patients that was correlated with more advanced $\mathrm{T}$ feature and more advanced clinical stage of cancer [16]. However, in the latter study no data about patients survival were provided and the analysis of the microphotographs presented in the latter study is difficult since the applied magnifications $(\times 10)$ do not allow for the exact assessment.

The meta-analyses of 20 IHC studies related to YAP1 presence in cancer cells showed that nuclear as well as overall (nuclear and cytoplasmic) localization of this protein correlated with poorer overall survival (OS) time and diseasefree survival time (DFS) in numerous types of cancer [24]. In the present study we demonstrated predominant nuclear YAP1 localization in ccRCC cells which may correspond to the YAP1's role as an oncoprotein which stimulates cell proliferation. Interestingly, the subgroup of ccRCC patients in whom cytoplasmic presence of YAP1 in cancer cells was observed exhibited the strongest correlation with patients' 
TABLE 2: Correlations between clinicopathological characteristics of the patients with clear cell renal cell carcinoma (ccRCC) and LATS1 immunoreactivity in the cancer cells (determined by immunohistochemistry).

(a)

\begin{tabular}{|c|c|c|c|c|}
\hline \multirow[b]{2}{*}{ Qualitative parameters } & \multirow{2}{*}{$\begin{array}{c}\text { Number of } \\
\text { patients }\end{array}$} & \multicolumn{3}{|c|}{ LATS1 immunoreactivity in ccRCC cells (expressed as IRS score) ${ }^{*}$} \\
\hline & & $\begin{array}{c}0-4 \\
n(\%)\end{array}$ & $\begin{array}{c}>4 \\
n(\%)\end{array}$ & $p$ value $^{\mathrm{a}}$ \\
\hline Total & 54 & $\begin{array}{c}22 \\
(40.7)\end{array}$ & $\begin{array}{c}32 \\
(59.3)\end{array}$ & \multirow{3}{*}{$1.0000^{\mathrm{a}}$} \\
\hline Men & 31 & $\begin{array}{c}13 \\
(41.9)\end{array}$ & $\begin{array}{c}18 \\
(58.1)\end{array}$ & \\
\hline Women & 23 & $\begin{array}{c}9 \\
(39.1)\end{array}$ & $\begin{array}{c}14 \\
(60.9)\end{array}$ & \\
\hline \multicolumn{5}{|l|}{ Depth of invasion } \\
\hline $\mathrm{T} 1+\mathrm{T} 2$ & 42 & $\begin{array}{c}15 \\
(35.7)\end{array}$ & $\begin{array}{c}27 \\
(64.3)\end{array}$ & \multirow{2}{*}{$0.1942^{\mathrm{a}}$} \\
\hline T3 & 12 & $\begin{array}{c}7 \\
(58.3)\end{array}$ & $\begin{array}{c}5 \\
(41.7)\end{array}$ & \\
\hline \multicolumn{5}{|l|}{ Fuhrman grade } \\
\hline $\mathrm{G} 1+\mathrm{G} 2$ & 42 & $\begin{array}{c}14 \\
(33.3)\end{array}$ & $\begin{array}{c}28 \\
(66.7)\end{array}$ & \multirow{2}{*}{$0.0507^{\mathrm{a}}$} \\
\hline G3 & 12 & $\begin{array}{c}8 \\
(66.7)\end{array}$ & $\begin{array}{c}4 \\
(33.3)\end{array}$ & \\
\hline \multicolumn{5}{|l|}{ Distant metastases } \\
\hline M0 & 47 & $\begin{array}{c}18 \\
(38.3)\end{array}$ & $\begin{array}{c}29 \\
(61.7)\end{array}$ & \multirow{2}{*}{$0.4260^{\mathrm{a}}$} \\
\hline M1 & 7 & $\begin{array}{c}4 \\
(57.1)\end{array}$ & $\begin{array}{c}3 \\
(42.9)\end{array}$ & \\
\hline
\end{tabular}

\begin{tabular}{lcr}
\hline Quantitative parameters & $r$ & $p$ value \\
\hline Age & 0.09 & $0.5370^{\mathrm{b}}$ \\
Tumor size & $-\mathbf{0 . 3 9}$ & $\mathbf{0 . 0 0 3 5}^{\mathrm{b}}$
\end{tabular}

${ }^{*}$ IRS: semiquantitative evaluation of immunoreactivity (percentage of cells $\times$ intensity score) was performed according to Remmele and Stegner [19] as described in Methods. ${ }^{\text {a } F i s h e r ~ e x a c t ~ t e s t . ~}{ }^{\text {b }}$ Spearman's correlation. RQ: relative quantification. Significant $p$ values $(<0.05)$ are given in bold.

poor prognosis. The Cox statistical test, independently of Kaplan-Meier analysis, revealed high hazard ratio of death of ccRCC patients demonstrating cytoplasmic YAP1 presence. This is the first prognostic observation of this kind that is of a potential importance for the assessment of the clinical status of the ccRCC patients. Our finding is supported by the results of several studies, which suggest that in the cytoplasm of cancer cells YAP1 may play a role as a molecular factor inducing more aggressive cancer behavior [25]. It has been shown that both YAP1 and KRAS have a common biding site which is the E2F transcription factor and that in the absence of KRAS signaling YAP1 functionally replaces KRAS in KRAS-dependent cancer cells [26]. It also is possible that not all of the cytoplasmic YAP1's molecules become degraded and some of them, or phosphorylated YAP1 protein, interact with the cell signaling pathways other than Hippo pathway that may stimulate proliferation of cancer cells. The oncogenic role of YAP1 may depend on the biology of primary cancer type. For instance, in liver tumors (hepatocellular carcinoma and cholangiocarcinoma) YAP1 cytoplasmic expression was associated with keratin 19 expression which was associated with cancer aggressiveness and patients' poor prognosis [27]. In squamous cell carcinoma of the uterine cervix the level of YAP cytoplasmic immunoreactivity was shown to be associated with histological grade, formation of lymph node metastasis, and cancer recurrence [28].

In our IHC study the presence of LATS1 was observed only within the cytoplasm of normal and cancer cells, although in $40 \%$ of patients no or weak immunoreactivity was found in ccRCC cells. Such localization corresponds to the well-known enzymatic function of LATS1 which phosphorylates and, thus, inactivates YAP1 protein in the cytoplasm [14]. In an immunohistochemical study lower LATS1 protein expression was found in renal cancer tissue and it correlated with the clinical stage and pathological grade of ccRCC [29]. Decreased level of LATS1 immunoreactivity in ccRCC cells, as observed in the present study, corresponds to the analysis of LATS1 gene expression on another set of ccRCC patients where we observed strong association between decreased LATS1 mRNA and protein contents in cancer tissue lysates [13]. We also identified the possible epigenetic mechanism of decreased LATS1 level: the hypermethylation of LATS1 
TABLE 3: Uni- and multivariate analysis (Cox proportional hazards regression) of overall survival of patients with clear cell renal cell carcinoma.

\begin{tabular}{|c|c|c|c|c|}
\hline \multirow{2}{*}{ Parameter } & \multicolumn{2}{|c|}{ Univariate Cox regression } & \multicolumn{2}{|c|}{ Multivariate Cox regression } \\
\hline & HR (95\% CI) & $p$ value & $\mathrm{HR}(95 \% \mathrm{CI})$ & $p$ value \\
\hline $\begin{array}{l}\text { YAP1 nuclear } \\
\text { immunoreactivity }\end{array}$ & $0.63(0.38-1.02)$ & 0.0625 & & \\
\hline $\begin{array}{l}\text { YAP1 cytoplasmic } \\
\text { immunoreactivity }\end{array}$ & $3.24(1.49-7.03)$ & 0.0029 & $4.53(1.73-11.9)$ & 0.0022 \\
\hline $\begin{array}{l}\text { LATS1 cytoplasmic } \\
\text { immunoreactivity }\end{array}$ & $0.90(0.81-0.99)$ & 0.0255 & $0.90(0.91-0.99)$ & 0.0289 \\
\hline $\begin{array}{l}\text { Gender } \\
\text { (women versus men) }\end{array}$ & $0.97(0.39-2.42)$ & 0.9525 & & \\
\hline $\begin{array}{l}\text { Age } \\
\text { (years) }\end{array}$ & $1.02(0.97-1.07)$ & 0.3958 & & \\
\hline $\begin{array}{l}\text { Tumor size } \\
(\mathrm{cm})\end{array}$ & $1.17(1.03-1.34)$ & 0.0168 & $1.12(0.93-1.36)$ & 0.2231 \\
\hline $\begin{array}{l}\text { Depth of invasion } \\
\text { (T3 versus } \mathrm{T} 1+\mathrm{T} 2 \text { ) }\end{array}$ & $2.03(0.77-5.37)$ & 0.1526 & & \\
\hline $\begin{array}{l}\text { Fuhrman grade } \\
\text { (G3 versus G1 + G2) }\end{array}$ & $4.07(1.60-10.4)$ & 0.0033 & $1.49(0.56-3.97)$ & 0.4299 \\
\hline $\begin{array}{l}\text { Distant metastases } \\
\text { (M1 versus M0) }\end{array}$ & $4.35(1.64-11.5)$ & 0.0032 & $4.94(1.59-15.3)$ & 0.0056 \\
\hline
\end{tabular}

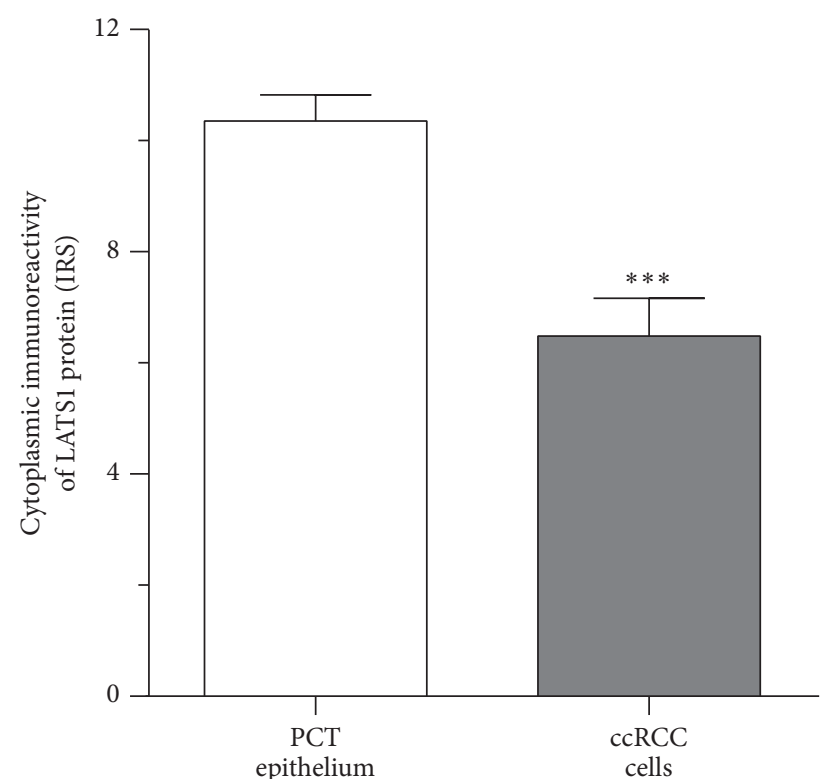

FIGURE 3: Comparison of serine/threonine-protein kinase 1(LATS1) immunoreactivity in the clear cell renal cell carcinoma (ccRCC) and unchanged kidney tissue. The level of cytoplasmic LATS1 immunoreactivity in tumor cells is shown in relation to its immunoreactivity in the epithelial cells of proximal convoluted tubules (PCT) of unchanged kidney. Data are presented as the mean $\pm \operatorname{SEM}(N=54) \cdot p$ values were calculated using the Wilcoxon matched-pair test. ${ }^{* * *} p<0.001$, as described in Methods.

promoter region was strongly associated with lower LATS1 content [13]. Although we did not check the association between LATS1 methylation and YAP1 protein content in our previous study, other authors in the study of ccRCC 786-O cell line observed that the demethylation of LATS1 promoter was strongly associated with upregulation of YAP1 protein in cells [29]. Since the relationship between epigenetic regulation of LATS1 (DNA methylation-mRNA-protein) and its influence on YAP1 protein cellular presence was verified, we suppose that IHC is a reliable method to assess LATS1 and YAP1 expression in ccRCC. Also the TCGA data obtained for 469 ccRCC tumors shows altered YAP1 mRNA and LATS1 mRNA level in cancer tumors. YAP1 mRNA was upregulated in $9.6 \%$ and downregulated in $4.9 \%$ cases and LATS1 mRNA was upregulated in $4.5 \%$ and downregulated in $10.7 \%$ cases, when a- $z$ score threshold was set for 1.5-fold change [30].

The present study lacks the statistical association of molecular data with the PFS observations due to insufficient outcome data of patients. However, there are only two studies that analyzed the influence of YAP1 expression on PFS in ovarian cancer (OC) [31] and colorectal cancer (CRC) [32]. It was shown that single-nucleotide polymorphism (SNP) which affects the upregulation of YAP1 gene was associated with OS and PFS in the study on 2901 OC as well as data of 4434 cases in TCGA [31]. The microarray study on 1028 patients with CRC indicated that high YAP1 mRNA levels were associated with shorter PFS and the upregulation of YAP1 mRNA was an independent prognostic factor of CRC $(\mathrm{HR}=1.82, p=0.034)$ [32]. Moreover, in the group of 36 mantle-cell lymphoma patients decreased LATS1 expression correlated with PFS and OS [33]. Such results cannot be compared with our data, since the expression of YAP1 and LATS genes was assessed at different levels: DNA [31] and mRNA $[32,33]$, whereas we analyzed the immunolocalization of protein products in the present study. 


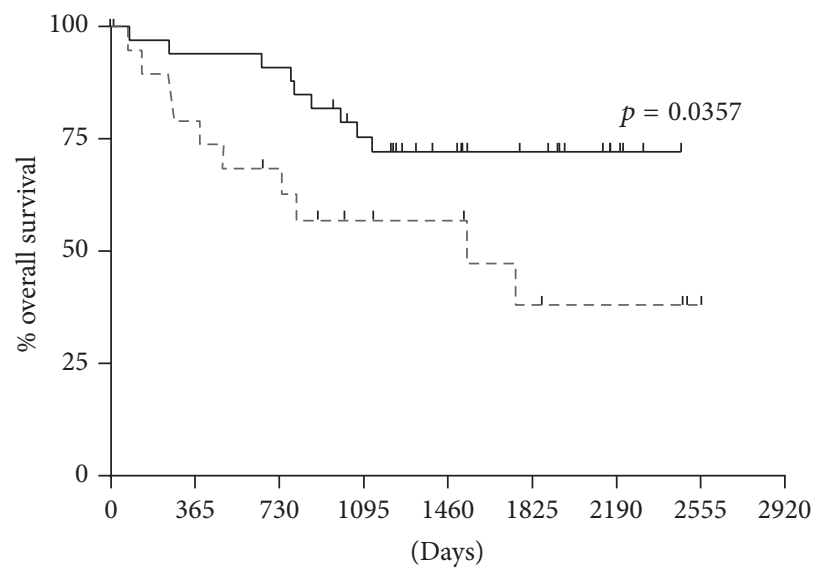

YAP1 nuclear immunoreactivity in ccRCC cells (score)

$-\perp-0-1$

$\perp 2-3$

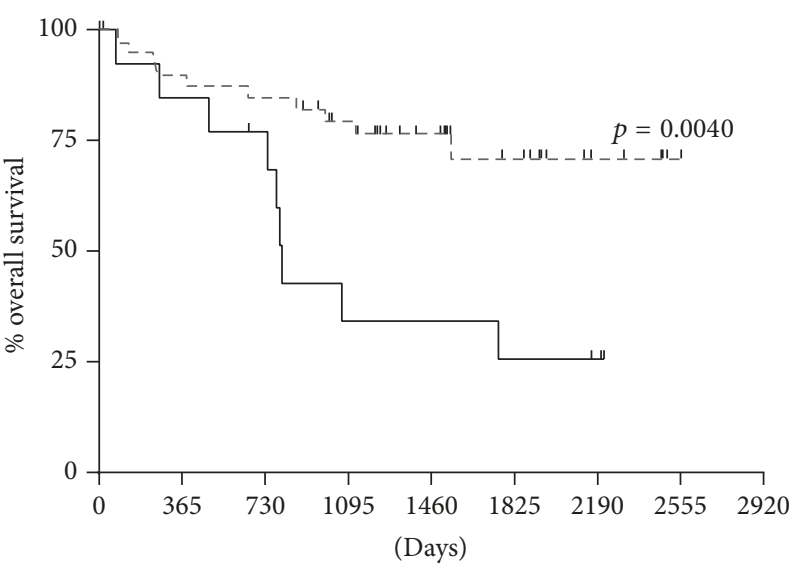

YAP1 cytoplasmic immunoreactivity in ccRCC cells (score)

$-1-0$

$\perp 1-2$

(a)

(b)

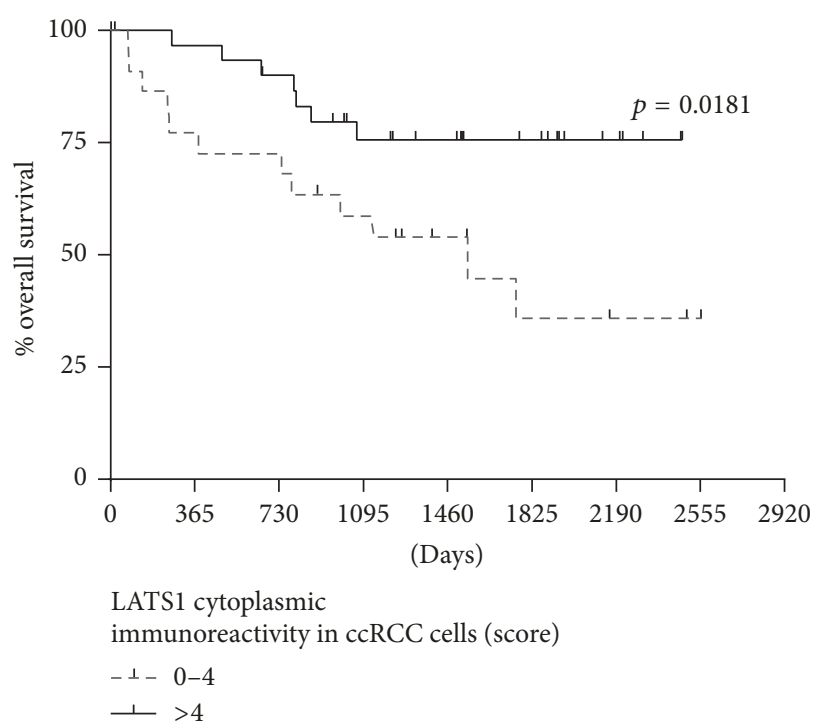

(c)

FIGURE 4: Analysis of overall survival of the patients. Kaplan-Meier curves of 54 ccRCC patients regarding the Yes-associated protein 1 (YAP1) nuclear (a), YAP1 cytoplasmic, (b) and serine/threonine-protein kinase 1 (LATS1) cytoplasmic (c) immunoreactivity levels. The scores of immunoreactivity were determined as described in Methods; $p$ values were calculated using the log-rank test.

\section{Conclusion}

The obtained results related to YAP1 and LATS1 immunoexpression in the cytoplasm of ccRCC cells suggest that immunohistochemical assessment of YAP1 and LATS1 status could be used as a prognostic marker and might be helpful in identifying high-risk ccRCC patients.

\section{Conflicts of Interest}

All authors declare no conflicts of interest.

\section{Acknowledgments}

The study was partially supported by National Science Centre (Poland) Grant 2012/05/B/NZ4/02735 and by statutory grants of the School of Medicine, Collegium Medicum, University of Warmia and Mazury in Olsztyn, Poland.

\section{References}

[1] J. Ferlay, I. Soerjomataram, R. Dikshit et al., "Cancer incidence and mortality worldwide: sources, methods and major patterns in GLOBOCAN 2012," International Journal of Cancer, 2014.

[2] B. Delahunt, "Advances and controversies in grading and staging of renal cell carcinoma," Modern Pathology, vol. 22, pp. S24S36, 2009.

[3] N. Bora-Singhal, J. Nguyen, C. Schaal et al., "YAP1 regulates OCT4 activity and SOX2 expression to facilitate self-renewal and vascular mimicry of stem-like cells," Stem Cells, vol. 33, no. 6, pp. 1705-1718, 2015. 
[4] J. A. Machado-Neto, M. Lazarini, P. Favaro et al., "ANKHD1, a novel component of the Hippo signaling pathway, promotes YAP1 activation and cell cycle progression in prostate cancer cells," Experimental Cell Research, vol. 324, no. 2, pp. 137-145, 2014.

[5] E. J. Vlug, R. A. H. Van De Ven, J. F. Vermeulen, P. Bult, P. J. Van Diest, and P. W. B. Derksen, "Nuclear localization of the transcriptional coactivator YAP is associated with invasive lobular breast cancer," Cellular Oncology, vol. 36, no. 5, pp. 375384, 2013.

[6] C. Zhu, L. Li, and B. Zhao, "The regulation and function of YAP transcription co-activator," Acta Biochimica et Biophysica Sinica, vol. 47, no. 1, pp. 16-28, 2015.

[7] T. Moroishi, C. G. Hansen, and K.-L. Guan, "The emerging roles of YAP and TAZ in cancer," Nature Reviews Cancer, vol. 15, no. 2, pp. 73-79, 2015.

[8] F. Zanconato, M. Cordenonsi, and S. Piccolo, "YAP/TAZ at the roots of cancer," Cancer Cell, vol. 29, no. 6, pp. 783-803, 2016.

[9] Y. Zhou, T. Huang, A. S. L. Cheng, J. Yu, W. Kang, and K. F. To, "The TEAD family and its oncogenic role in promoting tumorigenesis," International Journal of Molecular Sciences, vol. 17, no. 1, 2016.

[10] Z. Li, B. Zhao, P. Wang et al., "Structural insights into the YAP and TEAD complex," Genes \& Development, vol. 24, no. 3, pp. 235-240, 2010.

[11] Z. Meng, T. Moroishi, and K.-L. Guan, "Mechanisms of Hippo pathway regulation," Genes \& Development, vol. 30, no. 1, pp. $1-17,2016$.

[12] P. M. Wierzbicki and A. Rybarczyk, "The Hippo pathway in colorectal cancer," Folia Histochemica et Cytobiologica, vol. 53, no. 2, pp. 105-119, 2015.

[13] A. Rybarczyk, J. Klacz, A. Wronska, M. Matuszewski, Z. Kmiec, and P. M. Wierzbicki, "Overexpression of the YAP1 oncogene in clear cell renal cell carcinoma is associated with poor outcome," Oncology Reports, vol. 38, no. 1, pp. 427-439, 2017.

[14] S. Visser and X. Yang, "LATS tumor suppressor: A new governor of cellular homeostasis," Cell Cycle, vol. 9, no. 19, pp. 3892-3903, 2010.

[15] J.-J. Cao, X.-M. Zhao, D.-L. Wang et al., "YAP is overexpressed in clear cell renal cell carcinoma and its knockdown reduces cell proliferation and induces cell cycle arrest and apoptosis," Oncology Reports, vol. 32, no. 4, pp. 1594-1600, 2014.

[16] X. Hu, J. Chen, and Q. Fu, "Downregulation of YAP in clear cell carcinoma contributes to poor prognosis and progressive features," Annals of Clinical \& Laboratory Science, vol. 47, no. 1, pp. 36-39, 2017.

[17] I. D. Fleming, A. G. Fritz, C. M. Balch, D. G. Haller, and M. Morrow, AJCC Cancer Staging Manual, Springer-Verlag, Greene, FL, USA, 6th edition, 2002.

[18] S. A. Fuhrman, L. C. Lasky, and C. Limas, "Prognostic significance of morphologic parameters in renal cell carcinoma," The American Journal of Surgical Pathology, vol. 6, no. 7, pp. 655663, 1982.

[19] W. Remmele and H. E. Stegner, "Recommendation for uniform definition of an immunoreactive score (IRS) for immunohistochemical estrogen receptor detection (ER-ICA) in breast cancer tissue," Der Pathologe, vol. 8, no. 3, pp. 138-140, 1987.

[20] J. Godlewski, B. E. Krazinski, J. Kiezun et al., "PLAGL1 protein is differentially expressed in the nephron segments and collecting ducts in human kidney," Folia Histochemica et Cytobiologica, vol. 53, no. 1, pp. 96-104, 2015.
[21] Y. Wang, C. Xie, Q. Li, K. Xu, and E. Wang, "Clinical and prognostic significance of Yes-associated protein in colorectal cancer," Tumor Biology, vol. 34, no. 4, pp. 2169-2174, 2013.

[22] M. T. S. Allende, J. Zeron-Medina, J. Hernandez et al., "Overexpression of Yes Associated Protein 1, an Independent Prognostic Marker in Patients with Pancreatic Ductal Adenocarcinoma, Correlated with Liver Metastasis and Poor Prognosis," Pancreas, vol. 46, no. 7, pp. 913-920, 2017.

[23] X. Hu, Y. Xin, Y. Xiao, and J. Zhao, "Overexpression of YAP1 is Correlated with Progression, Metastasis and Poor Prognosis in Patients with Gastric Carcinoma," Pathology \& Oncology Research, vol. 20, no. 4, pp. 805-811, 2014.

[24] Z. Sun, R. Xu, X. Li et al., "Prognostic value of yes-associated protein 1 (YAP1) in various cancers: A meta-analysis," PLoS ONE, vol. 10, no. 8, Article ID e0135119, 2015.

[25] G. M. Sharif, M. O. Schmidt, C. Yi et al., "Cell growth density modulates cancer cell vascular invasion via Hippo pathway activity and CXCR2 signaling," Oncogene, vol. 34, no. 48, pp. 5879-5889, 2015.

[26] A. Kapoor, W. Yao, H. Ying et al., "Yap1 activation enables bypass of oncogenic KRAS addiction in pancreatic cancer," Cell, vol. 158, no. 1, pp. 185-197, 2014.

[27] K. Lee, K.-B. Lee, H. Y. Jung et al., "The correlation between poor prognosis and increased yes-associated protein 1 expression in keratin 19 expressing hepatocellular carcinomas and cholangiocarcinomas," BMC Cancer, vol. 17, no. 1, article no. 441, 2017.

[28] T. Liu, Y. Liu, H. Gao, F. Meng, S. Yang, and G. Lou, “Clinical significance of yes-associated protein overexpression in cervical carcinoma: The differential effects based on histotypes," International Journal of Gynecological Cancer, vol. 23, no. 4, pp. 735742, 2013.

[29] K.-H. Chen, J. He, D.-L. Wang et al., "Methylation-associated inactivation of LATS1 and its effect on demethylation or overexpression on YAP and cell biological function in human renal cell carcinoma," International Journal of Oncology, vol. 45, no. 6, pp. 2511-2521, 2014.

[30] Cancer Genome Atlas Research Network, "Comprehensive molecular characterization of clear cell renal cell carcinoma," Nature, vol. 499, no. 7456, pp. 43-49, 2013.

[31] S. E. Johnatty, J. P. Tyrer, S. Kar et al., "Genome-wide analysis identifies novel loci associated with ovarian cancer outcomes: findings from the ovarian cancer association consortium," Clinical Cancer Research, vol. 21, no. 23, pp. 5264-76, 2015.

[32] K.-W. Lee, S. S. Lee, S.-B. Kim et al., "Significant association of oncogene YAP1 with poor prognosis and cetuximab resistance in colorectal cancer patients," Clinical Cancer Research, vol. 21, no. 2, pp. 357-364, 2015.

[33] Z. Lu, S. Yi, Y. Zhang, Z. Li, and L. Qui, "Expression of LATS mRNA in mantle cell lymphoma and its clinical significance," Zhonghua Yi Xue Za Zhi, vol. 95, no. 40, pp. 3285-3288, 2015. 


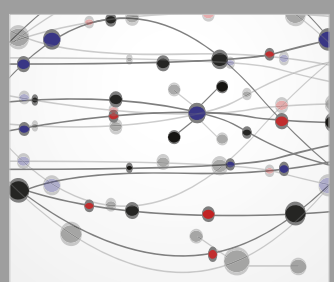

The Scientific World Journal
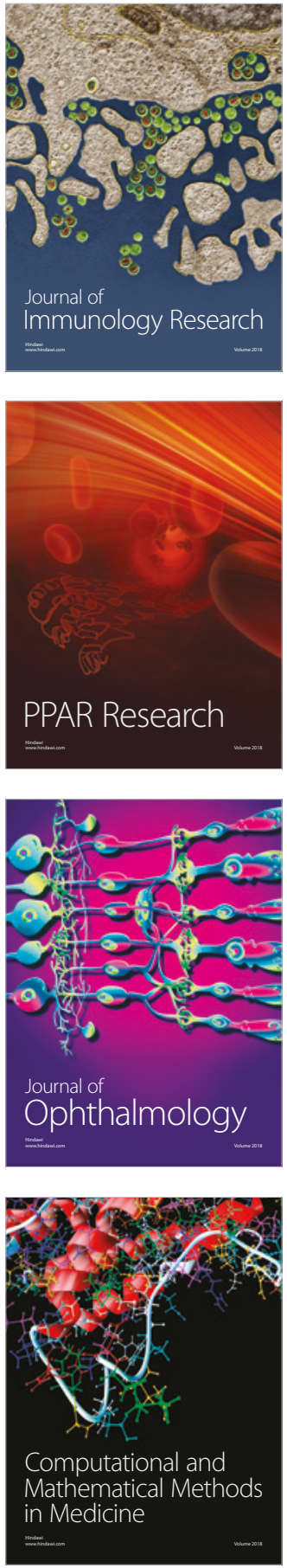

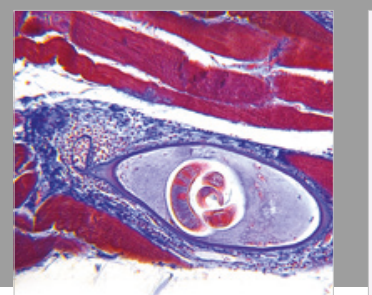

Gastroenterology Research and Practice

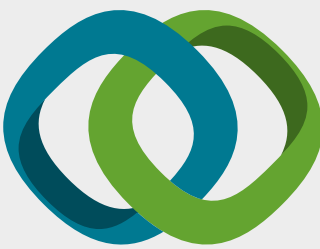

\section{Hindawi}

Submit your manuscripts at

www.hindawi.com
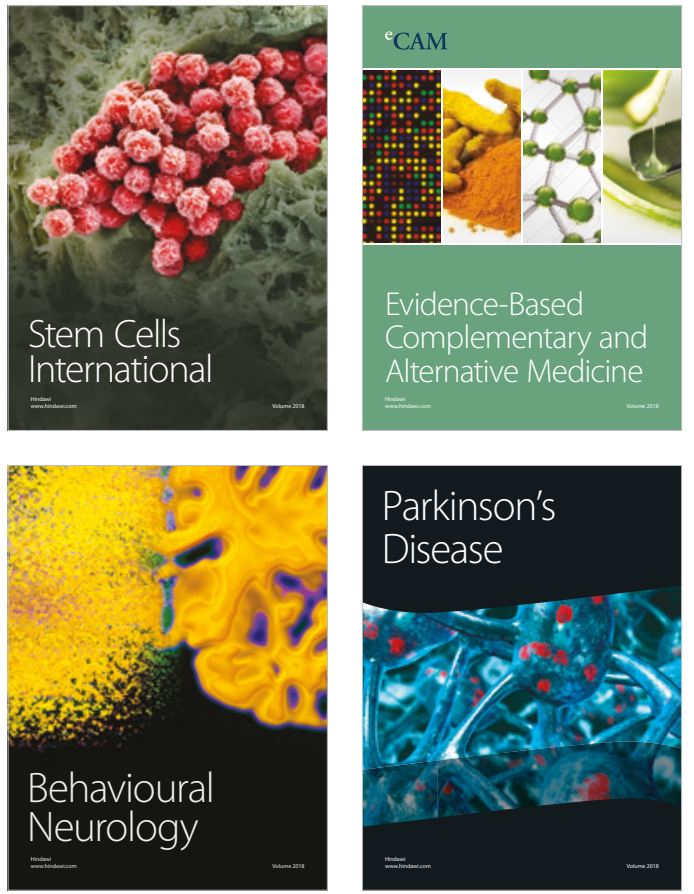

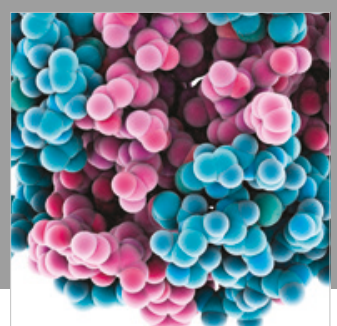

ournal of

Diabetes Research

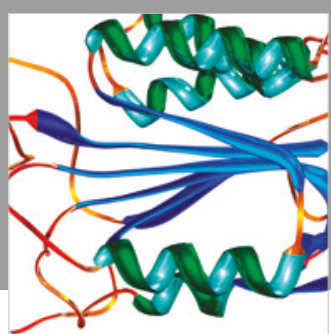

Disease Markers
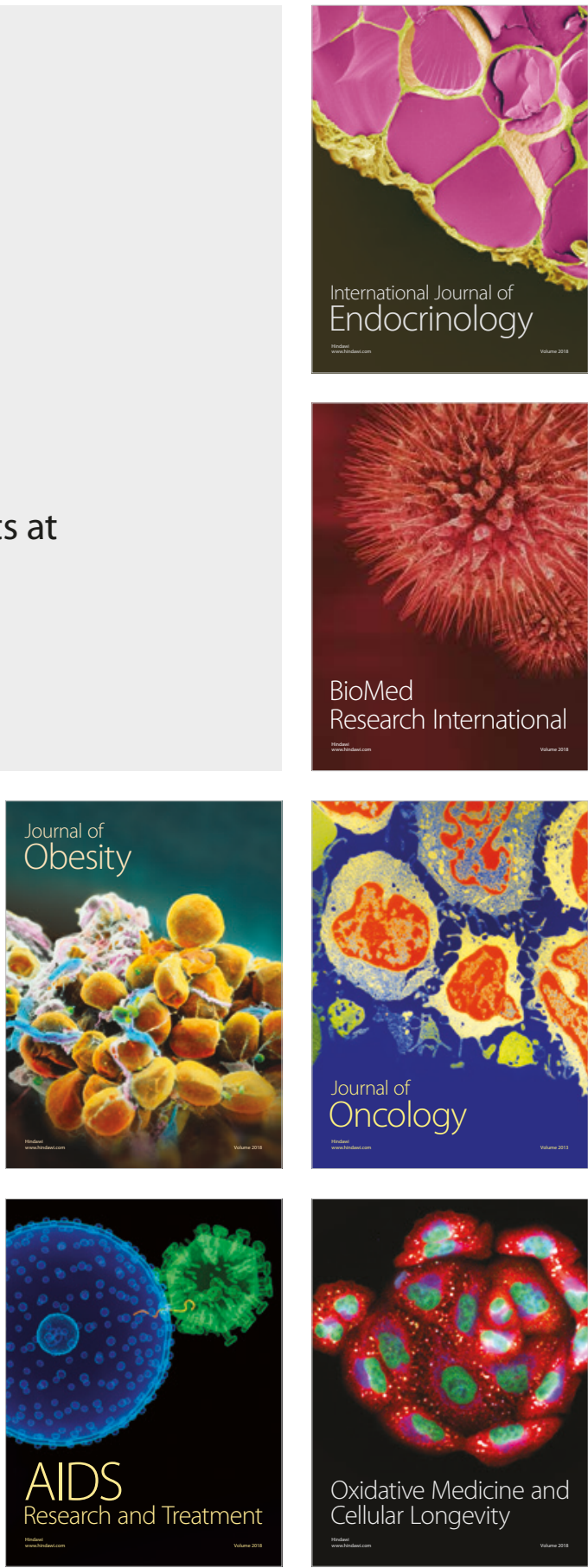